Cahiers de recherches médiévales

\title{
Dans le sillage de Jean le Bel
}

La chute d'Edouard II chez Jean d'Outremeuse et chez Froissart

Marie-Thérèse de Medeiros

\section{CpenEdition}

Journals

Édition électronique

URL : https://journals.openedition.org/crm/1603

DOI : $10.4000 / \mathrm{crm} .1603$

ISSN : 1955-2424

Éditeur

Honoré Champion

\section{Édition imprimée}

Date de publication : 15 mars 2003

Pagination : 131-142

ISSN : 1272-9752

Référence électronique

Marie-Thérèse de Medeiros, « Dans le sillage de Jean le Bel », Cahiers de recherches médiévales [En ligne], 10 | 2003, mis en ligne le 30 novembre 2009, consulté le 15 décembre 2022. URL : http:// journals.openedition.org/crm/1603; DOI : https://doi.org/10.4000/crm.1603

Ce document a été généré automatiquement le 15 décembre 2022.

Tous droits réservés 


\title{
Dans le sillage de Jean le Bel
}

\author{
La chute d'Edouard II chez Jean d'Outremeuse et chez Froissart
}

\author{
Marie-Thérèse de Medeiros
}

1 La Chronique de Jean le Bel, bien qu'elle ne nous ait été transmise que par un seul manuscrit, a certainement joui en son temps d'une solide notoriété locale, puisque c'est à elle que recourent, notamment pour relater la destitution du roi d'Angleterre Edouard II et l'avènement de son fils Edouard III, deux chroniqueurs nés environ un demi-siècle après le chanoine de Liège et originaires de la même aire géographique, Jean d'Outremeuse et Froissart ${ }^{1}$. C'est le rapport qu'entretiennent ces deux récits seconds au texte-source que j'aimerais examiner ici en m'attachant à l'insertion de cet épisode dans la vaste compilation que constitue le Myreur des histors ${ }^{2}$, puis à son traitement dans les trois versions parvenues jusqu'à nous des Chroniques.

2 En tête de son Myreur, Jean d'Outremeuse, alias Jean Des Prés, ne se présente pas en priorité comme un compilateur; il choisit un autre visage de l'homme de Lettres, traditionnel depuis le XII ${ }^{\mathrm{e}}$ siècle et le roman " antique ", celui du traducteur, du clerc qui, par sa connaissance du latin, met à la portée des laïcs des textes qui leur étaient jusqu'alors inaccessibles :

Portant que maintes gens oient volentirs racompteir en prendant solas et delectation en oyr parleir, racompteir, reciteir ou pronunchier anchienes hystors, croniques ou auctoritais et chozes anchienement passées et avenues le temps chidevant, nous Johans des Preis, dit d'Oultre-Mouse [...] vos vorons demonstreir et despondre chesty present croniques, que nos avons translateit de latien en franchois, et mys en pluseurs volummes ou libres dependant ly une del autre, affin que toutes manieres de singnour et aultres gens, qui de latien n'ont nulle cognissanche, le pussent entendre ${ }^{3}$.

On pourrait certes déjà voir dans cette déclaration liminaire se profiler l'ombre de la compilation, avec le travail d'agencement que laisse entendre la mention de parties «dependant ly une del autre ». Mais c'est seulement dans la phrase suivante du prologue, «Lyqueis presens croniques at esteit pris et extrais des croniques l'evesque de Liege Hue de Pirepont, les abbeis Engoran de Sains-Denys en Franche et Seguyn de Meauz en Brie... ${ }^{4}$, suivie de l'énumération d'une cinquantaine d'œuvres et d'auteurs que se manifeste cet autre savoir, lui aussi fort prisés au Moyen Âge, celui du 
compilateur, rappelé au terme de la présentation des sources: "Apres encor deveis savoir que, de tous ches croniques des maistres deseurdis, fut exstrais et copuleis chis presens croniques $»^{6}$. Je ne m'attarderai pas sur le fait que la Chronique de Jean le Bel s'insère mal, du point de vue de la langue, dans le corpus de Jean d'Outremeuse - ses compétences de traducteur n'étaient guère requises!- et m'en tiendrai à le voir à l'œuvre en tant que compilateur.

Un premier point peut d'emblée attirer l'attention du lecteur du Myreur des histors : la façon dont est amené le récit de Jean le Bel. En effet l'auteur ne se contente pas, comme bien souvent, de nommer sa source, il commence par présenter les circonstances dans lesquelles elle a vu le jour, donnant ainsi au récit qui va suivre un incontestable « label » de véracité. Ces circonstances sont bien connues de ceux qui s'intéressent à Jean le Bel et elles sont rappelées, entre autres, par Jules Viard et Nicole Chareyron? Ainsi, à supposer que Jean d'Outremeuse dise vrai, on a, grâce au compilateur, un éclairage sur le texte compilé, plus exactement sur les conditions de sa rédaction. Ce serait à la requête de Jean de Beaumont, frère du comte Guillaume de Hainaut et l'un des principaux soutiens de la reine Isabelle et de son fils - le futur Edouard III - que Jean le Bel aurait entrepris de composer sa Chronique, afin de dissiper les erreurs propagées, sur l'accession au pouvoir d'Edouard II et les débuts du conflit entre la France et l'Angleterre, par des « giestes qui contenoient mult grandes escriptures où ilh avoit pluseurs deffautes $»^{8}$. Un premier gage de vérité du récit de Jean le Bel tient à la situation de l'auteur qui a été témoin visuel (pas de meilleure garantie pour un esprit médiéval), comme le souligne l'information «qui presens avoit esteit... à tous les fais deseurdis $»^{9}$, mais il est encore renforcé par la constitution d'une sorte de comité de lecture dans lequel figurent "monsangnour Johans de Bealmont, le castelain de Waremme et plusors altres qui avoient esteit presens", capables de corriger d'éventuelles défaillances du chanoine de Liège ${ }^{10}$, puisque tous ont été "presens al fais $»^{11}$.

5 Une fois établi le caractère véridique de sa source, Jean d'Outremeuse en donne le contenu et va sans modifications majeures reproduire les XLI premiers chapitres de Jean le Bel, s'aventurant ainsi jusqu'en 1348, avant de reprendre son récit là où il l'avait interrompu pour insérer le récit de la Chronique, c'est-à-dire à l'année 1325 :

Je vuelhe d'or en avant revenir à ma matere de devant, ch'est de castelain de Waremme et des Flamens, apres le daute XIII ${ }^{c}$ et XXV chi-devant, là je le lassay à parleir d'on champ de batalhe que li castelain de Waremme deut avoir en Engleterre.... ${ }^{12}$

Quoiqu'il ait déclaré en introduisant le récit de Jean le Bel l'avoir mis dans son «s present croniques, nient tout (e) ensemble, mains la matere de cascon faite à le daute à chu afferantes, enssi qu'ilh appert chi-apres ", c'est d'un seul trait qu'il le livre à son lecteur, peut-être sensible à la séduction d'une narration qu'aurait rompu la présentation traditionnelle de la chronique passant en revue les différents événements du monde année par année. Il faut toutefois noter qu'il justifie chemin faisant cette infraction et précise, alors qu'il retrace, d'après Jean le Bel bien sûr, l'aventure du chevalier écossais «Guillaume » de Douglas ${ }^{13}$, chargé d'aller déposer le cœur de son roi en Terre sainte :

Quant li printemps vint et li bonne saison, sor l'ain XIIIc et XXVIII (car je vos vuelhe dire en ordine chesti mateire-chi jusqu'en la fin ou tou pres, por le mies entendre ou retenir et puis si retourneray à ma mateire sor l'ain XIIIc XXVII, et de là en avant 
enssi com vos oreis) si astoit mesire Guilheame proveus por paseir oultre le haulte meire enssi com à luy apartenoit... ${ }^{14}$

7 Si l'on en vient, après ces remarques d'ordre général sur l'insertion de la Chronique de Jean le Bel dans le Myreur des histors, à l'épisode relatant la chute d'Edouard II d'Angleterre, on ne peut que constater la fidélité du compilateur à sa source, avec une tendance à condenser on ne peut plus légitime, compte tenu de l'ampleur du champ d'investigation pris en compte par cette histoire universelle (du Déluge jusqu'au temps de Jean d'Outremeuse !). Le Myreur raconte donc, à la suite de Jean le Bel, comment le roi d'Angleterre Edouard II est tombé sous l'influence d'Hugues Spencer. Sur une dénonciation de son favori il fait exécuter certains grands barons du royaume, dont son oncle, le comte Thomas de Lancastre. Ces morts suscitent la haine de tout le pays à l'égard de Spencer, et en particulier celle de la reine, Isabelle, fille de Philippe le Bel, et celle du frère du roi, Edmond de Kent. Le favori riposte en faisant naître chez le roi une profonde aversion pour son épouse qui se réfugie en France auprès de son frère, le roi Charles IV, avec son fils aîné, Edouard; Edmond de Kent, Roger Mortimer et bon nombre d'autres chevaliers l'accompagnent. Edouard II multipliant les abus de pouvoir, les barons restés en Angleterre ainsi que l'ensemble du pays proposent à Isabelle de revenir avec une force armée et s'engagent à lui obéir. Ce projet est dans un premier temps soutenu par le roi de France, mais Hugues Spencer, informé du dit projet, gagne par des cadeaux et largesses l'appui des membres du conseil de Charles IV. Ce dernier défend alors à sa sœur de continuer ses préparatifs, et on laisse entendre à Isabelle de France que si elle ne se comporte pas «sagement $»^{15}$ elle sera renvoyée en Angleterre. Désemparée, la reine quitte la cour de France avec le jeune Edouard et tous ceux qui l'avaient accompagnée depuis l'Angleterre. Jean de Hainaut, seigneur de Beaumont, apprend qu'elle est hébergée en Cambrésis par un chevalier, il lui rend aussitôt visite et s'offre à soutenir sa cause, puis il la conduit ainsi que sa suite à Valenciennes chez son frère, le comte Guillaume de Hainaut. Jean de Beaumont rassemble un certain nombre de chevaliers en Hainaut, Brabant et Hesbaye et il gagne l'Angleterre avec ses compagnons, la reine, son fils et leur suite. Une tempête les empêche d'atteindre le port où ils pensaient aborder, ce qui est une véritable grâce du ciel ${ }^{16}$, car leurs ennemis, informés de leur arrivée les y attendaient. Quand le calme revient sur la mer ils gagnent la terre ferme et sont rapidement rejoints par ceux qui avaient fait appel à la reine. L'armée s'avance, chaque jour ralliée par de nouvelles forces et se dirige vers Bristol où se trouve le roi en compagnie d'Hugues Spencer, du père de ce dernier, du comte d'Arondel, de chevaliers et d'écuyers. L'armée assiège Bristol, la ville se rend, livre le père d'Hugues Spencer et le comte d'Arondel, qui sont exécutés. Edouard II et Spencer tentent de s'enfuir, mais ils sont rattrapés, Hugues Spencer est supplicié et le roi envoyé dans un château bien fortifié, « que je ne sçay nommer » déclare Jean le Bel, aveu d'ignorance que reproduit Jean d'Outremeuse. La plupart des compagnons de Jean de Beaumont regagnent alors leur pays, largement défrayés par la reine et son fils. Le jeune Edouard est couronné avec l'accord des seigneurs et des «bonnes villes » et Jean de Hainaut reste en Angleterre jusqu'à l'Épiphanie; mais quand il apprend qu'un tournoi organisé par le roi de Bohême et par son frère Guillaume de Hainaut va se tenir à Condé, le jeune roi et sa mère ne peuvent le retenir plus longtemps. La Chronique de Jean le Bel enchaîne ensuite sur le premier événement marquant du règne d'Edouard III, qui va ramener Jean de Beaumont en Angleterre : le défi lancé par le roi d'Ecosse au jeune souverain anglais, ce que fait également Ly Myreur. 
8 J'ai insisté sur la fidélité de Jean d'Outremeuse à l'égard de sa source et sur son choix en matière de reprise, l'abrègement. Il me faut pourtant signaler dans cet épisode un court passage, qui n'apparait pas chez Jean le Bel et que signalent Nicole Chareyron et Louis Gemenne $^{17}$ : il s'agit des sentiments d'Hugues Spencer à l'égard de la reine Isabelle. Après avoir, comme Jean le Bel, noté la responsabilité de Spencer dans la mésentente entre Edouard II et la reine, l'auteur du Myreur ajoute :

Et enamat Hue la damme, qui astoit I des plus belles damme qi fust en monde, et li requist de son amour : et elle li escondit; por quoy ilh le manechat et, apres le fist requerir d'altruy por avoir la connissanche charneile de lee : mains chis oit coupeit le chief, enssi que j'ay deviseit plainement deseur...18

«Enssi que j'ay deviseit plainement deseur »... le récit de la requête d'amour et de ses développements existe bel et bien, il se situe dans les pages qui précèdent les emprunts faits à la Chronique du chanoine de Liège, dans une première version de la chute d'Edouard II proposée au lecteur, à partir d'une source non identifiée, mais que S. Bormans verrait volontiers comme l'une de «ces hystoires rimées » dans lesquelles on «treuve [...] grand plenté de bourdes $»^{19}$ dénoncées par Jean le Bel dans son prologue. Sous la rubrique des événements de 1323 le Myreur note en effet :

En cel ain avient en Engleterre que Hue, li despensiers le roy, qui astoit li plus riches hons de tout le roialme, requist la damme d'amours, le femme le roy d'Engleterre, qui sœur astoit al roy Chairle de Franche et filhe à beal roy Philippe : dont la damme se corochat et ferit Hue si qu'ilh li brisat I dent. Adonc chis Hue, qui avoit bien $\mathrm{LX}^{\mathrm{m}}$ libres de revenue de terre par ain à l'escarlien, achat à I marchant le venien d'on serpent amerois et en donnat à boire le roy Eduart en Galles, où ilh estoit aleis, si que li roy enamat mult fort le despensiers par le forche de venien...20

10 On y trouve aussi l'étonnante mention d'un accouchement de la reine d'Angleterre entourée de la mère, la femme et la fille « del despensier » : l'enfant lui est enlevé puis noyé, tandis que l'on présente au roi «I pieche de chair ensanglantee » afin qu'il voie " queile fruit che estoit que sa damme avoit aporteit ». ${ }^{21}$ Toujours dans le registre du roman noir on voit un peu plus loin Spencer mettre en œuvre sa vengeance en dénonçant la reine au roi car il l'aurait trouvée «aveque I homme charnelment gesante ${ }^{22}$. Comme le roi refuse de croire que la fille du roi de France, sœur de trois rois puisse s'abaisser à de tels actes, Spencer propose une mise à l'épreuve. Que l'on envoie un beau chevalier auprès de la reine, qu'il soit porteur d'une lettre du roi recommandant à sa femme de faire pour ce chevalier tout ce qu'elle ferait pour lui en personne : on verra comment elle se comportera. La mission est confiée à un certain Guillaume " le chambrelain » à qui le favori du roi donne à boire « de venin del serpent et le instruit de male faire a chu que la royne ne posist escappeir ». Mais la reine, une fois la lettre lue, déclare qu'elle accorde tout "fors mis son corps et sa chambre". Comme le chevalier insiste et force l'entrée de la chambre, le jeune Edouard, qui se trouve près de sa mère s'interpose: le prétendant le gifle. Edmond de Kent (providentiellement présent!) coupe la tête du chevalier qui a osé manquer de respect au fils du roi. Mais Hugues Spencer sait tirer parti de cette mort et déclare que la reine a fait tuer le chevalier pour cacher son inconduite. Le roi se rallie à cette interprétation et la reine, pour sa sécurité, décide de gagner la France avec son fils, Edmond de Kent et quelques chevaliers. Soutenue dans un premier temps par son frère Charles IV, Isabelle est pour finir bannie de Francecar on l'accuse d'avoir voulu mettre le feu dans différentes villes du royaume. Les accusateurs, à qui Hugues Spencer a fait absorber « de venien de serpent amoreus en leur viandes » quant ils étaient en Angleterre, sont en fait des hommes à sa solde et le favori du roi a imaginé ce nouveau stratagème pour 
priver la reine d'appui. Comme chez Jean le Bel la reine trouve refuge auprès du comte de Hainaut et Jean de Beaumont lui offre son soutien, mande ses hommes et gagne l'Angleterre avec Isabelle de France et sa suite. On retrouve également le motif de la tempête, mais le merveilleux est ici plus explicite, puisque les éléments se déchainent à la prière de la dame, quand elle vient de reconnaitre sur un vaisseau les armes du comte d'Arondel (il appartient au parti favorable à Spencer et à Edouard II) et demande à Dieu de lui venir en aide si elle est dans son droit. Dans cette version les traitres, Arondel, Spencer sont également pris et suppliciés, mais les réactions de l'épouse d'Edouard II, ne s'harmonisent guère avec la dignité de son rang. Mise en présence du comte d'Arondel « la damme en demanda le cuer et elle le mangneroit si que cheli qui mult l'avoit greveit sens cause, par trahison ${ }^{23}$. Même requête à propos de Spencer, mais là le récit va plus loin et mentionne un quasi passage à l'acte : « et fut li cuer donneit à la royne, qui le wot mangier et l'eusse mordut quant ons li oistat $»^{24}$. La suite du récit lui réserve encore un rôle peu glorieux quand, cherchant désespérément un champion pour la défendre contre les accusations du comte de Vals, elle déclare :

«Hahay! roy, beals fis, je toy feray honte, car je promey à Dieu que se je trovoie I chevalier qui awist hardileche d'eistre mon champion, et ilh me vuelhe avoir, je m'en yray aveque li por faire sa volenteit; et se argent li falloit, je metteroie mon corps à cascon commonement à honte, por avoir del monoie à son talent $»^{25}$

11 Entre ses pulsions cannibales et sa facilité à envisager d'exercer le plus vieux métier du monde, la fille de Philippe le Bel n'est guère, on le voit, à la hauteur de son statut royal... mais déjà la dent brisée d'Hugues Spencer ne campait guère une reine en majesté.

invraisemblances et outrances de ce récit sont si nombreuses qu'il serait fastidieux de s'arrêter sur chacune d'entre elles. Je me contenterai de mentionner encore que lorsque le jeune Edouard est couronné avec l'accord des barons du royaume, cette décision a été prise « sor condition teile que là son peire li vies Eduart voroit reprendre sa meire en sa compangnie et faire raison, que ilh tout la court de sa vie ilh r'aroit la coronne ${ }^{26}$. Cette condition permet des développements abracadabrants sur une fausse réconciliation entre le roi et la reine, préparée par les ennemis d'Isabelle, une tentative de suicide du roi qui ne peut décidément plus vivre sans « Hue son despensier » et pour finir les accusations du comte de Vals contre lesquelles le châtelain de Waremme se propose pour défendre la reine. Pendant ce temps le comte de Hainaut s'étonne de ne pas voir revenir son frère et il l'envoie chercher en Angleterre. C'est pendant que les chargés de mission se trouvent à la cour du roi qu'arrive un messager qui annonce la mort d'Edouard II. Son fils se rend à Gloucester où il le fait enterrer avec tous les honneurs. Mais le bruit court que «cheaz qui le gardoient l'avoient mi à mort en I bangne et ochis par-desouz d'on chaut fier qu'ilh li butarent en cul à la base-chambre ou en bangne desus dite $\aleph^{27}$. Jean d'Outremeuse enchaîne alors sur la demande de Jean de Beaumont à Jean le Bel, non sans en avoir donné la raison : « et partant que chis fais fut si notoires par cristiniteit, si que fais de II roys tres-puissans, assavoir de roy de Franche et d'Engleterre, et partant les Engles (var. li juglieurs) fisent de chesti morteile guere giestes qui contenoient mult grandes escriptures, où ilh avoit pluseurs deffautes ".

On peut s'étonner avec S. Bormans ${ }^{28}$ de la démarche de Jean d'Outremeuse qui a fait précéder le récit de Jean le Bel d'une narration qui tient plus du conte de nourrice que de l'histoire. Mais présenter deux versions de certains faits est une pratique tout à fait digne d'un compilateur ${ }^{29}$ et l'auteur du Myreur avait bien précisé, après avoir présenté 
la première version du couronnement d'Edouard III, qui devait rendre le trône à son père si celui-ci se réconciliait avec la reine :

Et deveis savoir que li dit maistre Johans d'Oultre-Mouse eut et veit dois croniques parlant cascon de chest mateire, l'un contraire à l'autre : et vos en aperchureis en queis ch'est, car ilh les metit ensemble ${ }^{30}$.

De même il est de bon ton de laisser le lecteur se faire son opinion sur la validité des différentes versions, ce que fait ici Jean d'Outremeuse qui n'exprime pas ouvertement son choix, même si sa façon d'introduire le témoignage de Jean le Bel ne laisse aucun doute, on l'a vu plus haut, sur la véracité de son récit. Mais l'auteur du Myreur ne satisfait pas seulement à un usage, il laisse ici, une fois encore, affleurer son goût pour l'imaginaire, qui le pousse à intégrer dans sa chronique des éléments empruntés aux chansons de geste - Georges Doutrepont en mentionne plus d'une quinzaine ${ }^{31}$. Il lui arrive même à plusieurs reprises de renvoyer son lecteur, s'il veut en savoir davantage, à la geste (aujourd'hui perdue) qu'il a composée sur Ogier le Danois afin d'abréger son récit. «Mais de ce lairay esteit : je vous renvoye du tout a la gieste que j'en ay formee » déclare-t-il à propos des démêlés entre Charlemagne et Gérart de Fraite, ou encore, un peu plus loin, il se dispense de rapporter la bataille entre ogier et le roi sarrasin Brunaumont, l'ayant « escripte en la gieste Ogier, au plus prest de veriteit » et il ajoute

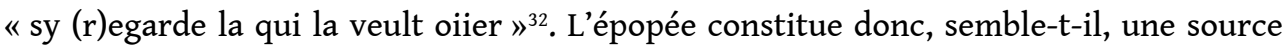
d'information tout aussi fiable que la chronique, et l'on peut comprendre dès lors que Jean d'Outremeuse juxtapose sans états d'âme la Chronique de Jean le Bel et un tissu d'invraisemblances, faisant la part belle à l'érotisme (les accusations portées contre la reine par Spencer ou le comte de Vals), à l'horreur (les pulsions cannibales d'Isabelle de France) ou à la magie (l'usage immodéré que fait Spencer du « venin de serpent »).

Tout autre est le rapport de Froissart au texte de Jean le Bel, auquel aucun autre récit ne vient s'opposer et qu'un certain nombre de détails ou de développements vient enrichir dans chacune des trois rédactions des Chroniques parvenues jusqu'à nous. Chaque rédaction présente des éléments différents et, comme il était prévisible, la version que propose le manuscrit de Rome (dernière rédaction) n'a plus qu'une très lointaine relation avec le texte originel, alors que la version du manuscrit $\mathrm{d}^{\prime} \mathrm{Amiens}^{33}$ et la version éditée en texte principal par la S. H. F. en restent assez proches. Froissart donc procède par amplification du récit du chanoine de Liège, et ce par une double voie, relevant aussi bien de «l'extension thématique» que de «l'expansion stylistique $»^{34}$.

16 Je m'attarderai peu sur les expansions qui relèvent du style et de l'art de « bien dire » et qui conduisent les Chroniques à développer une scène à peine esquissée, par Jean le Bel. Je retiendrai seulement, à titre d'exemple, l'arrivée en France de la reine d'Angleterre. Laconiquement le chanoine de Liège écrit : « et s'enfuit en France au roy Charles son frere, qui adonques regnoit $\aleph^{35} \mathrm{La}$ rencontre entre la reine d'Angleterre et le roi de France reste implicite et Jean le Bel enchaîne sur les abus de pouvoir d'Hugues Spencer et le mécontentement qu'ils suscitent en Angleterre. Froissart, lui, choisit de développer la séquence " arrivée de la reine en France » et en fait une scène courtoise et pleine d'émotion Le roi de France, voyant sa sœur " que grant tampz n'avoit veu " ${ }^{36}$ entrer dans la pièce où il se trouve, va à sa rencontre et lui souhaite la bienvenue à elle et à son fils ; puis la belle Isabelle de France expose ses malheurs à son frère, qui lui adresse des paroles de réconfort. On reconnaît dans cette expansion la manière de Froissart, son goût pour les scènes courtoises, ce goût qui lui fera reproduire dans ses trois rédactions l'épisode de Jean de Hainaut volant au secours de la reine d'Angleterre, 
dans le plus pur registre du chevalier et de la dame «desconseillée ». Expansion donc où se marque l'intérêt de Froissart pour l'écriture, et son penchant pour «l'emphase romanesque $\Perp^{37}$, au moins dans les deux premières rédactions. Dans le manuscrit de Rome, en effet, le récit de l'accueil de Charles IV est beaucoup plus succinct, l'embellissement romanesque moins perceptible, tandis que s'exprime le jugement du chroniqueur devant l'attitude de Charles IV, répondant aux doléances de sa sœur :

Et ordonna tantos de lor etat (celui de sa sœur, de son neveu et du comte de Kent), qant il ot entendu recorder sa serour et le conte de Qent la vie, l'affaire et l'ordenance dou roi d'Engleterre et de ce Hue le Espencier; mais il ne dist pas: «Belle serour, pour l'amour de vous, et pour ce que je voi que il se mesuse, je li manderai notorement que il se mete a raison et eslonge de li son mauvais consel et vous tiengne en paix et en estat, ensi que uns rois doit tenir sa femme, ou je li ferai guerre ». Nennil, mais li dist: « Ma belle serour, je vous pourverai courtoisement de vostre estat pour vous et pour vostre fil ; et entrués s'avisera vostres maris, ou li amour et la compagnie de li et de ce Hue le Espensier se desrompera ». Il convint la roine d'Engleterre prendre en bon gré ce que ses freres li rois de France li offroit, et l'en remercia et aussi fist li cntes de Qent ${ }^{38}$

Emblématique résignation de la reine d'Angleterre, qui est celle aussi du chroniqueur à qui l'expérience et le recul du temps ont appris que les grands de ce monde ne sont pas plus que les autres hommes des héros de roman...

Mais parallèlement à cette amplification par « bel langage " $^{39}$, qui n'apporte aucun fait nouveau par rapport au texte de Jean le Bel, cette séquence autour du départ d'Angleterre de la reine et de son arrivée en France s'enrichit de détails et précisions dont la fonction n'est plus du domaine de l'esthétique, mais du domaine de l'information et que j'ai rapprochés, bien que la correspondance ne soit pas parfaite, des « extensions » analysées par G. Genette ${ }^{40}$. C'est ainsi que les Chroniques s'attachent à l'itinéraire d'Isabelle de France et de sa suite et retiennent dans les trois versions l'arrivée à Boulogne des fugitifs puis leur marche vers Paris. Nombreuses sont les « extensions » de ce type chez Froissart. Notamment l'épisode du départ de France de la reine comporte un surcroît d'informations quand on le rapproche du texte-source. Alors que Jean le Bel se limitait à mentionner l'arrivée de la reine en Cambrésis où elle fut reçue « à l'ostel d'ung povre chevalier qui luy fist assez d'aise et de honneur selonc son petit pouoir $»^{41}$, demeure où Jean de Hainaut vint la rejoindre, Froissart ne fait plus du Cambrésis qu'un lieu de passage et nomme la localité où Isabelle fut hébergée ainsi que ses hôtes. L'identification est la même dans les trois versions, le château se trouve à «Buignicourt $»^{42}$, en Hainaut. Et il est habité par le seigneur "d'Aubrecicourt» (le « povre chevalier » de Jean le Bel sort ici de son anonymat!) ${ }^{43}$ et sa femme, qui gagnent par la courtoisie de leur accueil la reconnaissance et l'affection de la reine et de son fils.

On voit d'emblée l'origine de ce deuxième type d'ajouts de Froissart : ils tiennent à la recherche d'un supplément d'information, donc à une enquête du chroniqueur pour compléter les blancs laissés dans l'œuvre de son devancier. Ils témoignent donc de l'investissement d'un auteur qui, chaque fois qu'il le peut, essaie de saisir tous les éléments relatifs aux événements qu'il s'est donné pour mission d'enregistrer par l'écriture et par là d'arracher à l'oubli. Ils témoignent aussi en faveur de la justesse des propos de Froissart, en particulier dans le prologue du livre III ou celui du livre IV des Chroniques $^{44}$, où il se présente comme un insatiable quêteur d'information et de vérité. Cette dimension est très visible dans la troisième rédaction, où, comme je l'ai noté précédemment, le texte de Jean le Bel relatant la chute d'Edouard II n'est presque plus visible tant ont été modifiés les faits et les interprétations. 
20 Mais, sans entrer dans le détail de ces modifications, c'est sur une dernière information/«extension» que je souhaiterais achever ce propos. Il s'agit de l'incarcération et de la fin d'Edouard II. Jean le Bel, on l'a noté ci-dessus, déclare qu'il ignore en quel lieu a été emprisonné le père d'Edouard III et Jean d'Outremeuse reproduit ce constat d'ignorance. Chez Froissart en revanche les trois rédactions s'accordent pour nommer ce lieu de détention : " ung chastiel que on appelle Bercler », "ou fort chastiel de Bercler ", "ou chastiel de Bercler ", c'est-à-dire le château de Berkeley. Là s'arrête, comme chez Jean le Bel, dans les deux premières rédactions, le récit du sort de cet infortuné roi d'Angleterre. Toutefois le manuscrit de Rome poursuit :

Et ne vesqui puis le rois que il fu venus a Bercler, trop longement. Et conment euist il vesqu, par la maniere que je vous dirai ? Car je, Jehans Froissars acteres de ceste histore, fui ens ou chastiel de Bercler, l'an de grasce Nostre Signeur mille. CCC. LX. VI. ou mois de septembre, en la compagnie de messireEdouart le Espensier, liquels fu fils dou fil de ce messire Hue le Espensier, dont je parlerai assés tos; et fumes dedens le chastiel ens es esbatemens la environ, trois jours. Si demandai de che roi, pour justefiier mon histore, que il estoit devenus. Uns anciiens esquiers me dist que dedens le propre anee que il fu la amenés, il fu mors, car on li acourça sa vie. Ensi fina chils rois d'Engleterre.... ${ }^{45}$

21 On ne saurait souhaiter témoignage plus probant de la curiosité toujours en éveil de Froissart, de la passion qui le mène inlassablement à cerner, compléter « ceste haulte et noble hystoire » qui « sera en grant cours » alors que lui-même sera « mort et pourry ». 46

$\mathrm{Au}$ terme de cette confrontation ponctuelle entre Jean d'Outremeuse et Froissart l'auteur du Myreur des histors peut sembler faire piètre figure. Compilateur médiocre on est bien loin des savants agencements de sources que savent opérer par exemple les auteurs anonymes de l'Histoire ancienne jusqu'à César ou des Faits des Romains ${ }^{47}$ - s'il ne se distingue guère non plus par son discernement en reproduisant, à côté du récit de Jean le Bel, un récit aux multiples invraisemblances, belle préfiguration du roman noir. Toutefois on peut lui savoir gré d'avoir ainsi fait passer à la postérité un type de narration nourrie de racontars et de ragots que l'on pouvait considérer au Moyen Âge le Myreur en porte directement témoignage !-comme historique. Et, de ce point de vue, on peut penser que, comme Jacques Le Goff le souligne à propos du Ménestrel de Reims, il donne un aperçu sur les mentalités et «la consommation culturelle $»^{48}$ de son temps.

23 Ce qui ressort encore de la confrontation entre ces deux utilisateurs de la Chronique de Jean le Bel c'est la disparité de leurs sources. Alors que Froissart s'informe auprès de ceux qui ont vécu les événements qu'il se propose de relater (chevaliers, écuyers, hérauts d'armes ${ }^{49}$ ) Jean d'Outremeuse use de sources essentiellement livresques, ce qui se conçoit aisément pour l'élaboration d'une chronique universelle, mais qui demeure la règle du compilateur quand il se rapproche de son époque. De là peut-être son embarras quand il arrive au bout de ses sources et que s'achève son troisième livre :

Or vos ay deviseit trois libres de croniques ordineis par moy, Johain desus nommeis ; et si n'ay point de fin, ains convient le quart libre recommenchier, li queis ne serait mie fineis de mon temps ne à ma vie, mains ilh serait plus avant que je poray et sauray troveir croniques et mateire à cronisier ; car de mon temps de ma jovente je astoie jovene por cronisier, et quant je fuy devenus homme et je commenchay à estudoiier les histors et croniques, si les enamay mult : et fut dolens que je n'avoie cronisiet devant plus, al mains de temps que li croniques finoient de 
maistre Johan de Hoquesem et les croniques meisme mesire Johain Vestit de Warnant deleis Hui, qui sont de date del an XIIIc et XLVII del Nativiteit NostreSignour ${ }^{50} \ldots$

Pour Jean d'Outremeuse, on le voit, écrire un récit historique c'est « faire un livre avec des livres $»^{51}$, contrairement à Froissart pour qui c'est d'abord le fruit d'une enquête.

On pourrait, pour conclure, reprendre au sujet de l'auteur des Chroniques et de celui du Myreur l'opposition entre deux types d'historiens que François Hartog esquisse à propos de Polybe et de Timée. Jean d'Outremeuse, nouveau Timée, serait «l'historien en pantoufles et le rat de bibliothèque», celui qui se contente du "mol oreiller des voyages à travers les livres ", tandis que Froissart-Polybe, nouvel Ulysse, s'apparenterait à l'historien qui «doit avoir essuyé les embruns et fréquenté les champs de bataille ${ }^{52}$. Le chanoine de Chimay n'a sans doute pas hanté les lieux de combat, mais il a tiré ses informations de ceux qui s'y trouvaient, il le souligne à maintes reprises. Quant aux embruns essuyés, ils trouveraient aisément leur équivalent dans les voyages à cheval du chroniqueur, particulièrement dans le célèbre voyage en Béarn, entrepris, nous dit-il, " pour savoir la verité des lointaines marches, sans que je y envoiasse autre personne que moy $\aleph^{53}$. Primauté de la vision, de l'expérience directe, qui expliquerait peut-être, en partie ${ }^{54}$, le choix de Froissart retenant la chronique du chanoine de Liège pour les années les plus reculées, là où l'information lui faisait défaut. C'est aussi, probablement, la justification des compléments et modifications apportés à l'hypotexte. Pourtant c'est à Jean d'Outremeuse que revient le mérite d'avoir insisté sur la qualité du récit de Jean le Bel, tenant à son rôle d'acteur et de témoin. Et l'on risque fort de ne jamais savoir si l'auteur du Myreur avait/aurait rallié, pour les événements contemporains la méthode de ses compatriotes dans le livre IV de son ouvrage, celui-ci étant perdu... ou n'ayant jamais existé ${ }^{55}$.

\section{NOTES}

1. Froissart est, on le sait, né à Valenciennes, Jean d'Outremeuse à Liège, le premier vers 1337 , le second en 1338.

2. Les références renverront à l'édition d'A. Borgnet et S. Bormans, Ly Myreur des histors, Bruxelles, 1864-1880, en six volumes.

3. Ly Myreur, I, pp. 1-2.

4. Ibid., pp. 2-4.

5. Cf., notamment, B. Guenée, "L'historien et la compilation au XIII ${ }^{\mathrm{e}}$ siècle », Journal des Savants, 1985, pp.119-135; L'historiographie médiévale en Europe, éd. par J. Ph. Genet, Paris, 1991, pp. 21-116 ; C. Croizy-Naquet, Écrire l'histoire romaine au début du XIII ${ }^{e}$ siècle., Paris, 2000.

6. Ly Mireur, p. 4.

7. Jules Viard, Introduction à la Chronique de Jean le Bel, éd. J. Viard et E. Déprez, Paris, 1904-1905, vol. I, pp. VIII-X ; Nicole Chareyron, Jean le Bel. Le maître de Froissart, Grand Imagier de la guerre de Cent Ans, Bruxelles, 1996, pp. 52-53.

8. Ly Myreur, VI, p. 322.

9. Ibid., pp. 322-323. 
10. On pourrait considérer que cette dénomination s'applique aussi à Jean d'Outremeuse. Mais je me range au point de vue de $\mathrm{S}$. Bormans qui considère que L'auteur du Myreur n'était pas nécessairement chanoine et que les emplois dans son récit d'un " je » renvoyant à un chanoine sont dûs au fait que J. d'O. reproduit ce qui est écrit dans sa source sans l'adapter en troisième personne. On peut également observer ce trait à propos de la reproduction du récit de la campagne d'Écosse où Jean le Bel use de la première personne en tant qu'acteur dans cette campagne et où le compilateur conserve les «je » et les «nous ». Froissart, plus rigoureux, adapte en troisième personne.

11. Ly Mireur, VI, p. 323.

12. Ibid., p. 392. La partie compilée de la Chronique de Jean le Bel se situe p 323-388 du vol. VI de l'édition Borgnet-Bormans, elle correspond à ce que $\mathrm{J}$. Viard considère être la première phase de composition de l'œuvre.

13. Son prénom était Jacques.

14. Ly Mireur, VI, pp. 349-350.

15. Jean le Bel, I, p. 13, Ly Myreur, VI, p. 328.

16. Jean le Bel, « de quoy Dieu leur fist grande grace », I, p. 18, Ly Myreur, « et Dieu le fist por le melhour ", VI, p. 330.

17. N. Chareyron, op. cit., pp. 46-47, L. Gemenne, «Trois auteurs en quête de texte : les débuts de la guerre de Cent Ans selon Jean le Bel, Jean Froissart et Jean d'Outremeuse ", dans Convergences médiévales. Épopée, lyrique, roman. Mélanges offerts à M. Tyssens,éd. N. Henrard, P. Moreno, M. ThiryStassin, Bruxelles, 2001, p. 177.

18. Ly Myreur, VI, p. 326.

19. Ly Myreur, VI, p. 326, note 6. N. Chareyron reprend, mais sur le mode de l'hypothétique cette proposition, op. cit, p. 46

20. Ibid., p. 270.

21. Ibid., p. 272.

22. Ibid., p. 301.

23. Ibid., p. 308.

24. Ibid., p. 310.

25. Ibid., p. 320. C'est en revenant à cette séquence de la reine en quête de champion et à l'engagement du châtelain de Waremme que Jean d'Outremeuse reprend son récit, après avoir reproduit la Chronique de Jean le Bel, VI, p. 392, cité infra.

26. Ibid., p. 314.

27. Ibid., p. 321.

28. Chronique et geste de Jean des Preis dit d'Outremeuse. Introduction et table des matiéres, Bruxelles, 1887, p. CLXI.

29. Cf. B. Guenée, Histoire et culture historique dans l'Occident médiéval, Paris, 1980, p. 130.

30. Ly Myreur, p. 314-315.

31. Les Mises en prose des Epopées et des Romans chevaleresques. Du XIVe au XVI siècle, Bruxelles, 1939, pp. 165-167.

32. Ly myreur des histors. Fragment du second livre. (Années 794-826), éd. A. Goosse, Bruxelles, 1965, p. 3, p. 57-58.

33. À partir des travaux de J. J. N. Palmer, G. T. Diller, P. F. Ainsworth, on considère actuellement que la première version est celle du ms d'Amiens, la seconde celle éditée par la S. H. F.

34. On aura reconnules deux composantes de l'amplification que retient Gérard Genette dans Palimpsestes, 1982, pp. 298-313. Il faut toutefois noter que les « extensions » des textes de Froissart dans les passages retenus ne procèdent pas par "addition massive ", comme l'extension des textes littéraires retenus par G. Genette.

35. Jean le Bel, op. cit., p. 10-11. 
36. Chroniques, le manuscrit d'Amiens, éd. G. T. Diller, Genève, 1991, I, p.11. Expression quasi identique pour la seconde version, Chroniques, Livre I et livre II, éd. P. F. Ainsworth et G. T. Diller, Paris, 2001, p. 84.

37. Michel Zink, Froissart et le temps, Paris, 1998, p. 61.

38. Chroniques. Début du premier livre. Edition du manuscrit de Rome Reg. Lat. 869, éd. G. T. Diller, Genève, 1972, p. 50.

39. L'expression se retrouve fréquemment chez Froissart et notamment pour désigner son travail d'écriture. Cf. notamment tome XII de l'édition de la S. H. F., p. 3.

40. On pourrait justifier le rapprochement des informations précises et des «extensions » par le fait que dans les deux cas il y a augmentation de la matière, alors que dans le cas des expansions ce qui domine, c'est « une sorte de dilatation stylistique », G. Genette, op. cit., p. 304.

41. Op. cit., p. 14.

42. Ms d'Amiens, éd. G. T. Diller, I, p. 19 ; seconde rédaction, éd. P. F. Ainsworth, G. T. Diller, p. 89 ; ms de Rome, éd. G. T. Diller, p. 58. Les informations de le Bel et Froissart semblent diverger, mais le ms de Rome, beaucoup plus détaillé, fournit une explication : la reine et sa suite ont bien été d'abord accueillis en Cambrésis par le seigneur d'Esne, mais c'est à Bugnicourt que Jean de Hainaut viendra retrouver la reine et se mettra à son service. Deux versions partielles que vient concilier une version plus large.

43. Ms d'Amiens, I, p. 19 ; seconde rédaction, p. 89 ; ms de Rome, p. 58.

44. Éd. S. H. F., tome XII, pp. 1-2, pour le livre III. Ed. Kervyn de Lettenhove, tome XIV, pp. 1-2, pour le livre IV.

45. Ms de Rome, op. cit., p. 90. La date donnée ici par Froissart pose problème, car la critique s'accorde sur une rédaction postérieure à 1366 pour les deux premières rédactions des Chroniques. Faut-il penser que lors de ces deux premières rédaction du livre I le chroniqueur confronté à l'ampleur du champ chronologique des événements retracés, de 1325 à 1378 environ, ne s'est pas attardé sur un point qu'il considérait comme secondaire? En revanche, ce complément d'information trouvait doublement sa place dans une récriture plus limitée dans le temps 1325-1350 - et dans un récit où s'affiche la présence de l'auteur.

46. Éd. S. H. F., tome XII, p. 2.

47. Voir C. Croizy-Naquet, Écrire l'histoire romaine..., op. cit., pp. 108-113.

48. Jacques Le Goff, Saint Louis, Paris, 1996, p. 377. Le rapprochement avec les Récits d'un ménestrel de Reims (éd. N. de Wailly, Paris, 1876, voir aussi la traduction avec commentaires de M. G. Grossel, P. U. de Valenciennes, 2002) peut se justifier à partir d'anecdotes où la dignité royale est passablement malmenée, comme celle qui relate le suicide d'Henri II Plantagenêt dans des latrines ou celle de Blanche de Castille, soupçonnée d'être enceinte et se dénudant devant prélats et vassaux pour démentir l'accusation.

49. Voir par exemple éd. Ainsworth-Diller, p. 71. Cf. aussi à propos de la fin d'Edouard II le témoignage de l'« anciien (s) esquier (s) » cité ci-dessus.

50. Myreur, VI, p. 637.

51. Cf. B. Guenée, "L'historien et la compilation au XIII ${ }^{\mathrm{e}}$ siècle », op. cit., p. 120. La formule est " faire des livres avec des livres", et l'auteur donne son origine: A. Momigliano, Problèmes d'historiographie ancienne et moderne, Paris, 1983, p. 29.

52. F. Hartog, Mémoire d'Ulysse. Récits sur la frontière en Grèce ancienne, Paris, 1996, p. 176.

53. Éd. S. H. F., tome XII, p. 2.

54. Il est évident qu'ont joué également la notoriété locale, comme je l'ai souligné en introduction, et tout autant la qualité littéraire de la chronique de Jean le Bel.

55. Voir l'introduction de S. Bormans, Chronique et geste de Jean des Preis dit d'Outremeuse..., op. cit. 


\section{AUTEUR}

MARIE-THÉRÈSE DE MEDEIROS

Université d'Orléans 